\title{
Uveitis, diabetes, or cataracts? The mysterious case of the blurry left eye
}

\author{
Laura A. Goldberg* \\ Wilmington VA Medical Center, 1601 Kirkwood Hwy,Wilmington, DE 19805, USA
}

\begin{abstract}
Uveitis and diabetes can lead to macular edema and cataracts, resulting in reduced vision. This report discusses a unique case history of a patient presenting with all of these ocular pathologies, in which we were challenged to determine the cause of ocular inflammation and take a stepwise approach to managing each condition. Through utilization of clinical reasoning and ancillary testing, we were able to identify cataracts as the primary cause of reduced vision. The patient's chief complaint was successfully resolved with a treatment regimen of high-dosage topical steroid and cycloplegic agent.
\end{abstract}

\section{Introduction}

\section{Initial visit}

A 67-year-old African-American male was sent to the eye clinic from the emergency room secondary to waking up to a sharp pain (scale 9/10, 1 being the least painful, 10 being the most painful) four days ago with redness and light sensitivity left eye (OS), clear right eye (OD). Patient reported he used lubricating eye drops with no relief. Patient stated that the pain started to subside throughout the day without any further medical intervention. The next two mornings he awoke with slight discharge/pus in eye with itchiness. The night before coming to the emergency room he experienced the worst pain so far (10/10) with itchiness/irritation, prompting him to immediately visit the emergency room at VA Hospital. ER performed computed tomography (CT) maxillofacial scan without contrast prior to coming to eye clinic to rule out any sinusitis. No associated pathology was found. Patient was given erythromycin ointment to be used on as-needed basis both eyes (OU) and Percocet every 6 hours orally. Upon initial visit at eye clinic patient was still experiencing eye pain and light sensitivity $(8 / 10)$ but no subjective changes in vision per patient.

\section{Systemic history}

Patient has previously been diagnosed with type II diabetes. Patient reported he discontinued metformin a year ago by personal decision. Patient has essential hypertension with history of poor compliance (last blood pressure reading was 159/80). Patient has hyperlipidemia with no updated meds/lab tests available. Patient has history of coronary arteriosclerosis for which he was currently being treated with $81 \mathrm{mg}$ aspirin daily. Patient's social history demonstrated no tobacco, alcohol or recreational drug abuse.

\section{Background on last exam}

Last appointment was two years ago. Patient was diagnosed with diabetic (not clinically significant) macular edema with mild diabetic retinopathy both eyes (OU). Vision was reduced OS, best corrected visual acuity (BCVA) was 20/30. Patient reported trauma as a child OS; however, patient was poor historian. Previous chart noted physiologically large cupping OU.

\section{Entrance testing}

BCVA was 20/20 OD; 20/150 with no improvement pinhole (PH) OS. Modified potential acuity meter (mPAM) measured 20/20 OD, 20/50 OS. Pupil testing showed a slowly reactive pupil OS but no evidence of afferent pupillary defect (APD). Extraocular muscles (EOMS) and confrontation fields (CF) were full.

\section{Slit lamp exam}

Lids demonstrated mild meibomian gland dysfunction (MGD). Conjunctiva showed trace injection OD and 2+ hyperemia (denser peri-limbal) OS. Sclera was clear OU. Cornea showed mild corneal edema OS with diffuse superficial punctate keratitis (SPK) inferiorly OS greater than OD and no evidence of keratic precipitates (KPs) OU. Iris was flat OU with no evidence of neovascularization of iris (NVI) / angle recession (AR). Anterior chamber was deep and quiet OD, 4+ cells/2+ flare reaction OS with no hypopyon OU. Lens showed 1+ nuclear sclerosis (NS) OD and 2+ NS OS. Goldmann Applanation Tonometry (GAT) measured $18 \mathrm{OD}$ and 15 OS at 9:52 am. Gonioscopy was open to ciliary body band (CBB) 360 with iris processes $\mathrm{OU}$ and no evidence of angle recession (AR) or peripheral anterior synechiae (PAS).

\section{Fundus exam findings}

Optic nerve head cup-to-disc ratio was $0.65 / 0.70 \mathrm{OD}, 0.65 / 0.70$ OS with healthy rim tissue and no evidence of pallor OU. Macula demonstrated few microaneurysms (MAs) parafoveal OD and OS with no evidence of clinically significant macular edema (CSME) OU. Vitreous had only mild syneresis with no vitritis OU. Periphery was clear and intact with no evidence of neovascularization, vasculitis, or phlebitis OU.

Correspondence to: Laura A. Goldberg, Wilmington VA Medical Center, 1601 Kirkwood Hwy,Wilmington, DE 19805, USA, E-mail: laura.goldberg@va.gov

Key words: Uveitis, macular edema, diabetic retinopathy, cataracts

Received: May 24, 2017; Accepted: June 14, 2017; Published: June 17, 2017 


\section{Medication administered}

Patient was given 1 drop (gtt) of tropicamide 1\% OD; 1 gtt homatropine 5\% and $1 \mathrm{gtt}$ prednisolone acetate $1 \%$ OS at 10:20 am.

\section{Additional testing}

Optical Coherence Tomography (OCT) - Macular and (HighDefinition) HD 5-line raster at exam two years prior showed mild diabetic macular edema at foveal center OD, minimal cystic edema near fovea OS. Macular OCT at present exam showed slightly abnormal foveal contour due to small pocket of intra-retinal fluid superior to fovea OS only (Figures 1,2).

\section{Differential diagnoses considered}

\section{Anterior segment}

1) Bacterial conjunctivitis

2) Corneal abrasion

3) Dry eye syndrome

4) Acute anterior unilateral non-granulomatous uveitis OS

\section{Posterior segment}

5) Macular edema secondary to mild non-proliferative diabetic retinopathy vs. anterior uveitis spillover

\section{Anterior segment}

- Bacterial conjunctivitis does result in irritation and sometimes light sensitivity and shows signs of redness and discharge. However, patient did not give any indication of having a recent cold. Furthermore, it is often found in younger population, is bilateral, and results in more copious discharge. Also, important to note is that erythromycin ointment did not show any effect on resolving symptoms/signs.
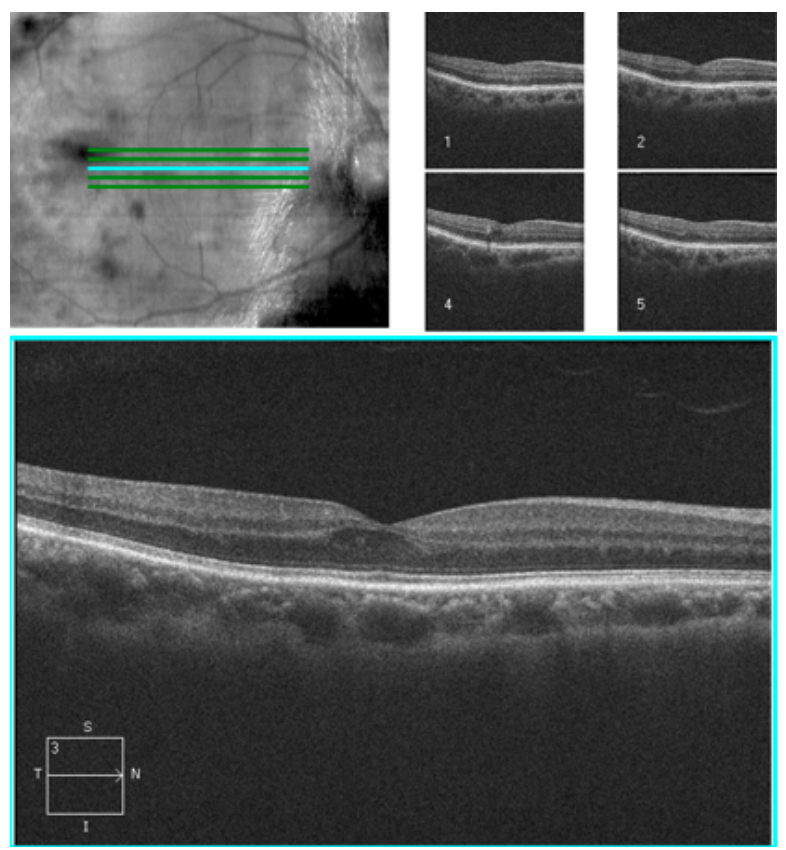

A
- Corneal abrasion can result in unilateral extreme pain, tearing and light sensitivity. However, fluorescein staining only demonstrated of mild SPK OU, no epithelial defect.

- Dry eye syndrome can result in foreign body sensation, irritation, sharp pain, and light sensitivity. SLE showed diffuse SPK which was symmetrical OD and OS. Patient, however, only reported symptoms OS and lubricating eye drops previously applied by patient did not help to relieve symptoms.

- Acute anterior unilateral non-granulomatous uveitis often is acute onset with moderate pain and significant amount of cells and flare with marked perilimbal injection.

\section{Posterior segment}

- Diabetic edema secondary to diabetic retinopathy is a reasonable differential diagnosis due to patient's ocular history of mild diabetic retinopathy with macular edema OU.

- Cystoid macular edema (CME) secondary to spillover of uveitis is commonly seen in patients, especially with heavy grade of inflammation in anterior chamber and unilateral presentation.

\section{Initial diagnosis}

Due to acute onset, hyperemic conjunctiva, intense inflammation of anterior chamber, no KPs noted and sluggish pupil OS, it was reasonable to determine that this case represented anterior uveitis. Furthermore, based on MAs near fovea OU, patient was also labeled as mild non-proliferative diabetic retinopathy. The etiology of the macular edema was yet to be determined.

Upon questioning, patient stated no lower back pain worse in the morning, no bad cough, no oral/genital sores, no history of being in woods or tick bites and no difficulty with bowel movements. Lab testing was ordered the same day including Lyme titer, enzymelinked immunosorbent assay (ELISA) for herpes simplex virus, chest
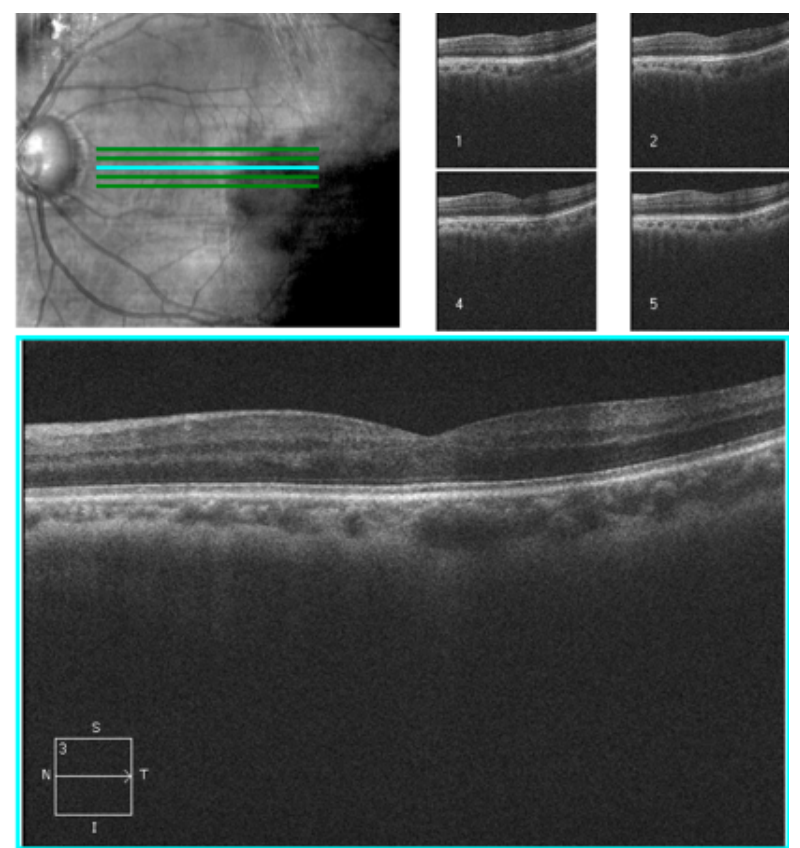

B

Figure 1. HD 5-line raster two years prior with cross-section through macula A) Right eye B) Left eye. Right eye shows small pocket of cystoid macular edema, left eye normal foveal contour with minimal edema. 
ODILII-RPE THCKiess UIP

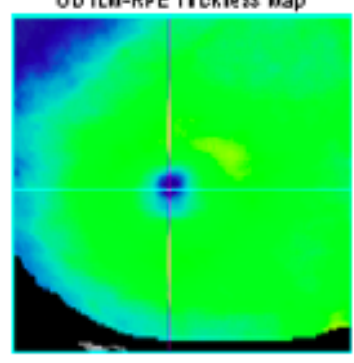

Fovea: 239,66

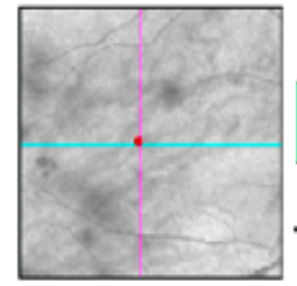

OO LLI-RPETH Lkiets

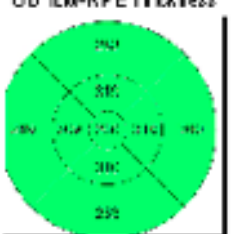

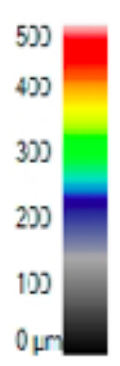

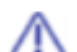

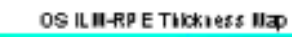

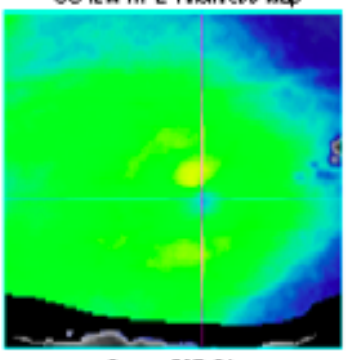

Fouea: 298,71
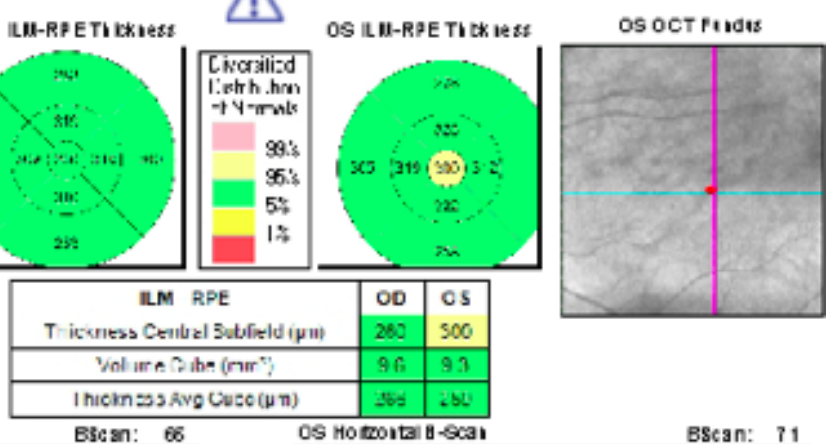

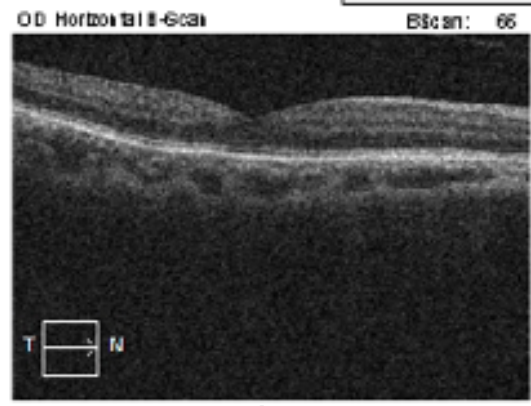

A

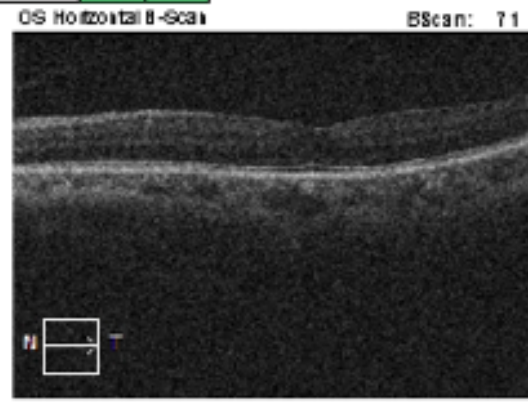

B

Figure 2. Macular OCT from initial visit with normal macular thickness A) Right eye and B) Left eye. Note increased central macular thickness indicated by yellow color marker.

radiograph (for sarcoidosis/ tuberculosis), purified protein derivative (PPD), angiotensin-converting enzyme (ACE) level, rapid plasma regain (RPR), fluorescent treponemal antibody absorption (FTA-ABS), human leukocyte antigen B27 (HLA-B27), erythrocyte sedimentation rate (ESR), and C-reactive protein (CRP). Patient was also directed to use prednisolone acetate $1 \%$ every hour $(\mathrm{Q} 1 \mathrm{H})$, homatropine $2 \mathrm{x}$ day (BID) OS and phenylephrine $2.5 \% 2 \mathrm{x}$ day (BID) OS and to return to clinic four days later.

\section{Follow-up 1 (4 days after initial visit)}

Lab testing was positive for HLA-B27. Patient reported strict compliance with all medication as well as $99 \%$ relief of signs (redness) and symptoms (light sensitivity and irritation). BCVA was 20/20- OD, 20/70 PH 20/60+ OS. Entrance testing was same as initial visit; pupil testing showed pharmacologically dilated pupil OS with no reverse APD. Slit lamp examination (SLE) indicated resolution of lid swelling OS, 1+ diffuse hyperemia OS, mild SPK OU. Anterior chamber showed $2+$ cells/ 1+ flare OS. GAT measured 15 OD, 14 OS at 11:23 am. Fundus exam was same as initial visit. Patient was informed that he was positive for HLA-B27. Sacroiliac (SI) joint fusion test was ordered due to patient's age and history of mild arthritis to rule out ankylosing spondylitis.

We educated the patient on the importance of prompt and aggressive treatment due to the potential risk of secondary complications, such as glaucoma, and high recurrence rates of uveitis in patients with HLA-B27 and spondyloarthropathies. Patient was directed to continue with prednisolone acetate 1\% Q1H and homatropine 5\% BID OS and discontinue phenylephrine $2.5 \%$ BID OS for next two weeks until follow-up.

\section{Follow-up 2 ( 3 weeks after initial visit)}

Patient admitted to taking prednisolone acetate $1 \%$ every 2 or 3 hours instead of every hour and was compliant with homatropine 5\% BID OS. BCVA today was 20/20-2 OD and 20/40 PH 20/40+ OS. Pupils, EOMS and CF were same as previous visits. GAT was $21 \mathrm{OD}$, 22 OS at 10:52 am. SLE showed complete resolution of edema OS, mild SPK OU. Anterior chamber was deep and quiet OD, trace (3) cells/flare OS. Due to improvement of symptoms/signs but not full resolution of inflammation OS, patient was instructed to continue with 
prednisolone acetate $1 \%$ every 2 hours $(\mathrm{Q} 2 \mathrm{H})$ and homatropine $5 \%$ BID OS. Patient was scheduled for a two-week follow-up visit.

\section{Follow-up 3 (5 weeks after initial visit)}

Patient stated that he was taking prednisolone acetate 1\% QID OS and homatropine 5\% BID OS. BCVA 20/20-2 OD and 20/50+2 PH NI mPAM 20/25+ OS. Pupils, EOMS and CF were same as previous visits. GAT measured $22 \mathrm{OD}, 22 \mathrm{OS}$ at 8:24 am. SLE showed complete resolution of corneal edema OS, mild SPK OU. Anterior chamber was deep and quiet OD, trace cells/flare OS. Due to stable anterior chamber reaction patient was told to taper prednisolone acetate $1 \% 3 x$ day for 5 days, then $2 \mathrm{x}$ day for 5 days, then $1 \mathrm{x}$ day until follow-up and discontinue homatropine.

\section{Telephone Contact (7 weeks after initial visit)}

After case review with attending, patient was started on diclofenac $4 \mathrm{x}$ a day (QID) OS secondary to mild retinal edema OS. When patient returned for follow-up visit in two weeks, OCT would then be performed again to monitor for resolution of edema with topical NSAID treatment OS.

\section{Follow-up 4 ( 9 weeks after initial visit)}

Patient reported tapering of prednisolone acetate $1 \% 1 \mathrm{x}$ per day (QD) and taking diclofenac QID OS. Patient stated he was started on metformin per primary-care physician (no glucose or blood testing performed yet). BCVA was 20/25-2, PH 20/20-3, mPAM 20/20- OD, 20/50-2 PH 20/40+ mPAM 20/20- OS. Entrance testing and pupils were same as first follow-up visit. Cover test was normal, four baseout test was negative. Retinoscopy demonstrated symmetrical mild hyperopia with astigmatism. BCVA 20/20 OD and 20/50+2 OS. SLE was same as last visit with anterior chamber deep and quiet $O D$, trace cells (3) OS. GAT was 19 OD, 19 OS at 10:15 am. Fundus exam was stable from initial exam with few MAs surrounding fovea OU. OCT findings showed mild pocket of intra-retinal fluid OS with stable
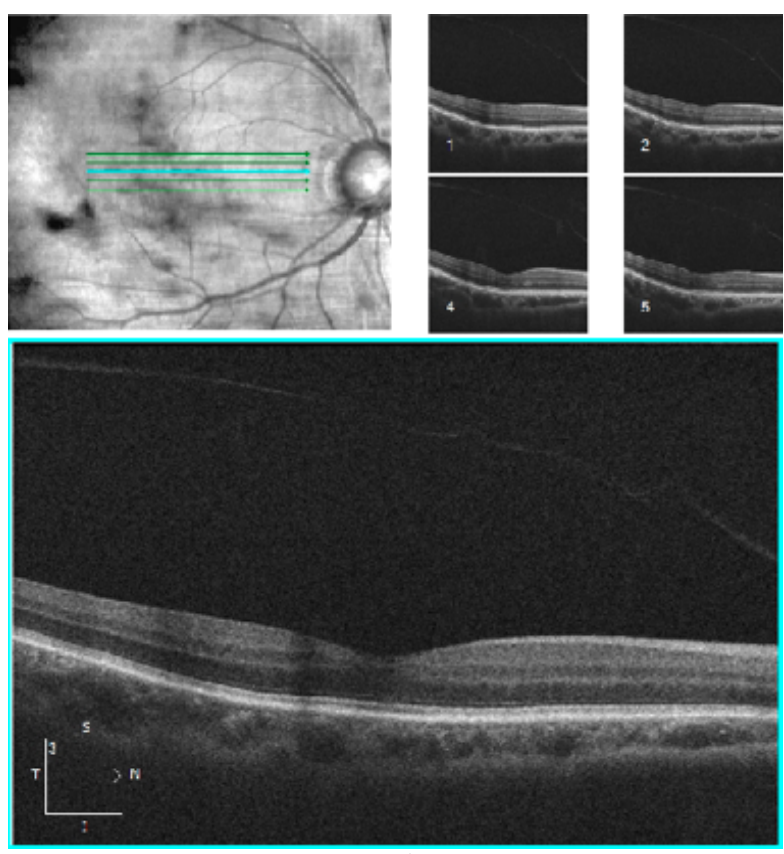

A findings comparing thickness values (Figure 3). Symmetrical refraction and no evidence of traumatic optic neuropathy on retinal nerve fiber layer (RNFL) OCT (Figure 4) ruled out amblyopia or trauma as cause of reduced vision OS.

The chief ophthalmologist was consulted who recommended increasing dosage of prednisolone acetate $1 \%$ to QID OS and continue diclofenac QID OS in attempt to completely resolve macular edema.

Lastly, SI joint fusion test was performed and results indicated "mild to moderate sacroiliac joint degenerative change with no definitive erosive arthropathy". We discussed the results with the patient and ordered consult for follow-up with rheumatology in one week and follow-up with eye clinic in four weeks.

\section{Follow-up 5 (13 weeks after initial visit)}

Upon review of records at visit, rheumatology confirmed the HLA-B27 diagnosis and ruled out all rheumatologic causes of the acute uveitis episode OS. Patient reported he has been using prednisolone acetate 1\% QID OS and diclofenac QID OS. Lab testing had been updated in the last month indicating $\mathrm{HbAlc}$ level of 15.4; no fasting blood sugar levels available per patient.

BCVA was 20/25-2, PH 20/20-2, mPAM 20/20-2 OD, 20/60+ PH 20/40+2 mPAM 20/20- OS with new habitual Rx updated from last exam. Entrance testing and pupil testing were same as first follow-up visit. SLE was same as last visit. Anterior chamber deep OD/OS. GAT measured $14 \mathrm{OD}, 15 \mathrm{OS}$ at 11:10 am. Fundus exam was stable from initial exam. OCT findings showed mild pocket of intra-retinal fluid OS stable from last follow-up.

The chief ophthalmologist was again consulted who agreed that patient should begin to taper prednisolone acetate $1 \%$. Diclofenac was not helping to resolve macular edema and therefore discontinued. Patient was scheduled for intravenous fluorescein angiography (IVFA) in one week. Patient was scheduled to see retinal specialist the following week.
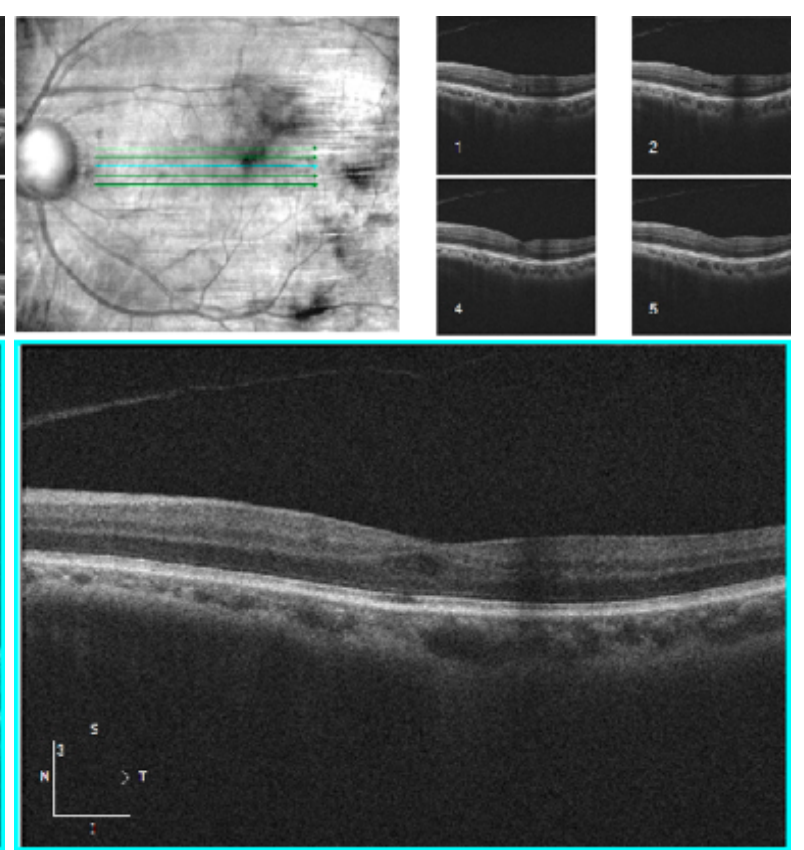

B

Figure 3. HD 5-line raster with cross-section through macula A) Right eye: normal foveal contour B) Left eye: mild cystic space superior temporal to fovea. 


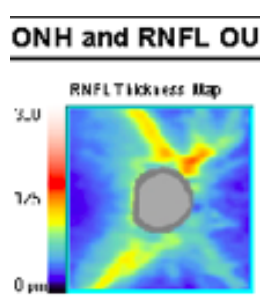

RNFL Deu Ltos Itap
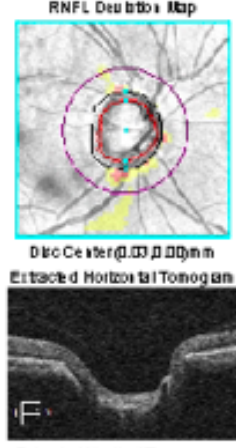

Extrated Vertal Tonogan
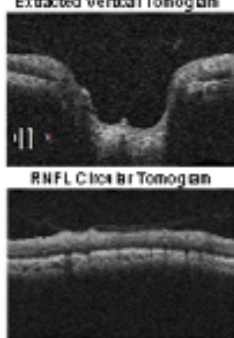

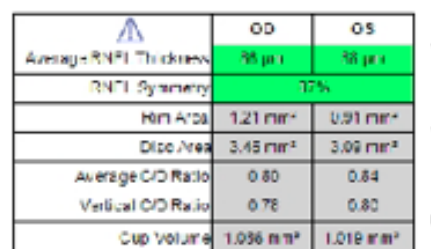

(10)
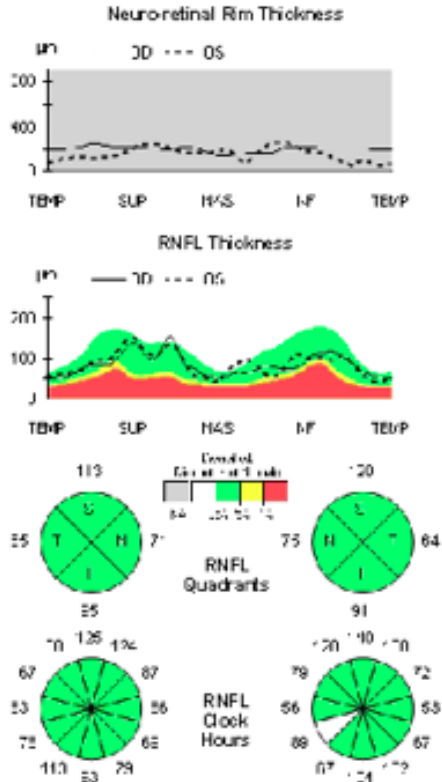

91

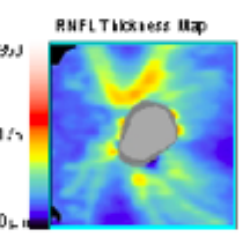

RNFL DeU LBOS ItaP

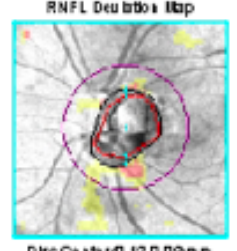

Deroce iter(10.12, D D g nn Etact d Hortzoutal Tonogran
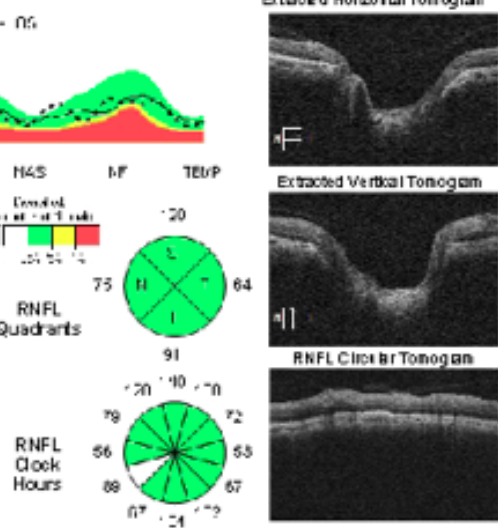

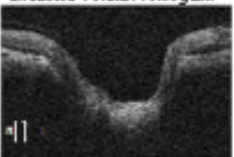
REFLCIEA BrTanogan

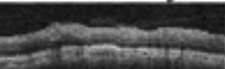

A

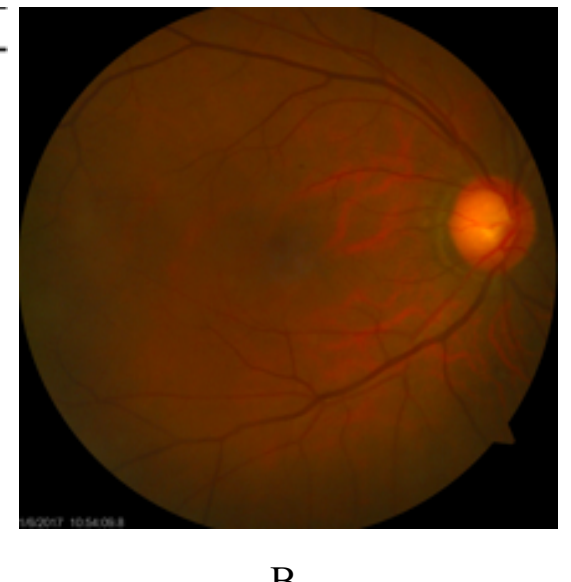

$\mathrm{B}$

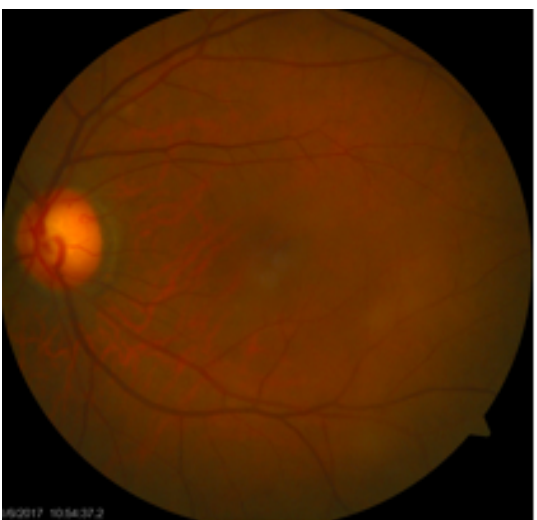

Figure 4. RNFL OCT of optic nerves with corresponding fundus photos A) Right eye B) Left eye. No evidence of glaucomatous thinning 360 OU.

\section{Follow-up 6 (14 weeks after initial visit)}

Results of IVFA demonstrated parafoveal MAs with minimal late leakage OU. Patient was confirmed to follow-up in a week with retina specialist for further review and assessment including whether treatment was indicated (Figure 5).

\section{Follow-up 7 (15 weeks after initial visit)}

Patient was seen by retina specialist who agreed with findings presented above. Specialist ascertained that cystic intra-retinal fluid was minimally affecting BCVA OS and reduced vision likely due to cataract. Quality of OCT and IVFA photos was insufficient for definitive diagnosis; however, no evidence of foveal ischemia or atrophy was found. Patient was instructed to start tapering prednisolone acetate $1 \%$, discontinue drops in one week and return to clinic in four weeks to monitor.

\section{Discussion}

\section{Uveitis and HLA-B27}

This ocular condition can be caused by a myriad of systemic disorders including autoimmune disorders, infection, malignancy, or just be idiopathic in nature. Approximately $50 \%$ of cases are due to idiopathic conditions, $20 \%$ due to trauma, $20 \%$ due to an underlying systemic issue and $10 \%$ due to localized ocular conditions such as herpes [1]. Therefore, it is important to take a good case history and perform comprehensive lab testing in order to rule out any systemic causes.
Anterior uveitis comprises $60 \%$ to $90 \%$ of all reported cases, with idiopathic causes being the most common of anterior uveitis. Furthermore, approximately 50\% are associated with the HLA-B27 allele [2]. Approximately $1 \%$ of people who are HLA-B27 positive develop acute anterior uveitis and $55 \%$ of cases of acute anterior uveitis are associated with an HLA-B27 positive serotype [3,4]. In addition, $84 \%$ of HLA-B27 positive patients with acute anterior uveitis have other B27-associated diseases; specifically Reiter's syndrome, ankylosing spondylitis or psoriatic arthritis [5].

There exist conflicting data on whether or not the presence of HLA-B27 influences recurrence rates. Norn (1969) found the overall recurrence rate in their subjects to be $55 \%$, and all with associated arthritis had $100 \%$ recurrence [6]. Power et al. (1998) determined that presence of spondyloarthropathy with HLA-B27 was associated with higher relapse rates than for those who were HLA-B27 positive alone, or HLA-B27 negative [7]. However, Linssen et al et al. (1991) reported no increase in recurrence with HLA-B27. These contradictory results may be attributable to variability in patient populations and/or severity of uveitis [3].

Studies have shown that HLA-B27 positive patients had more severe inflammation, more recurrences, longer duration, a higher complication rate, and worse visual outcome (11\% of patients with visual acuity less than 20/200 compared to $2 \%$ in B27 negative patients) than patients with idiopathic uveitis. Complications included extensive, persistent synechiae, glaucoma secondary to the inflammation itself or to the use of topical steroids, vitritis, papillitis, and cystoid macular edema [7-9]. 

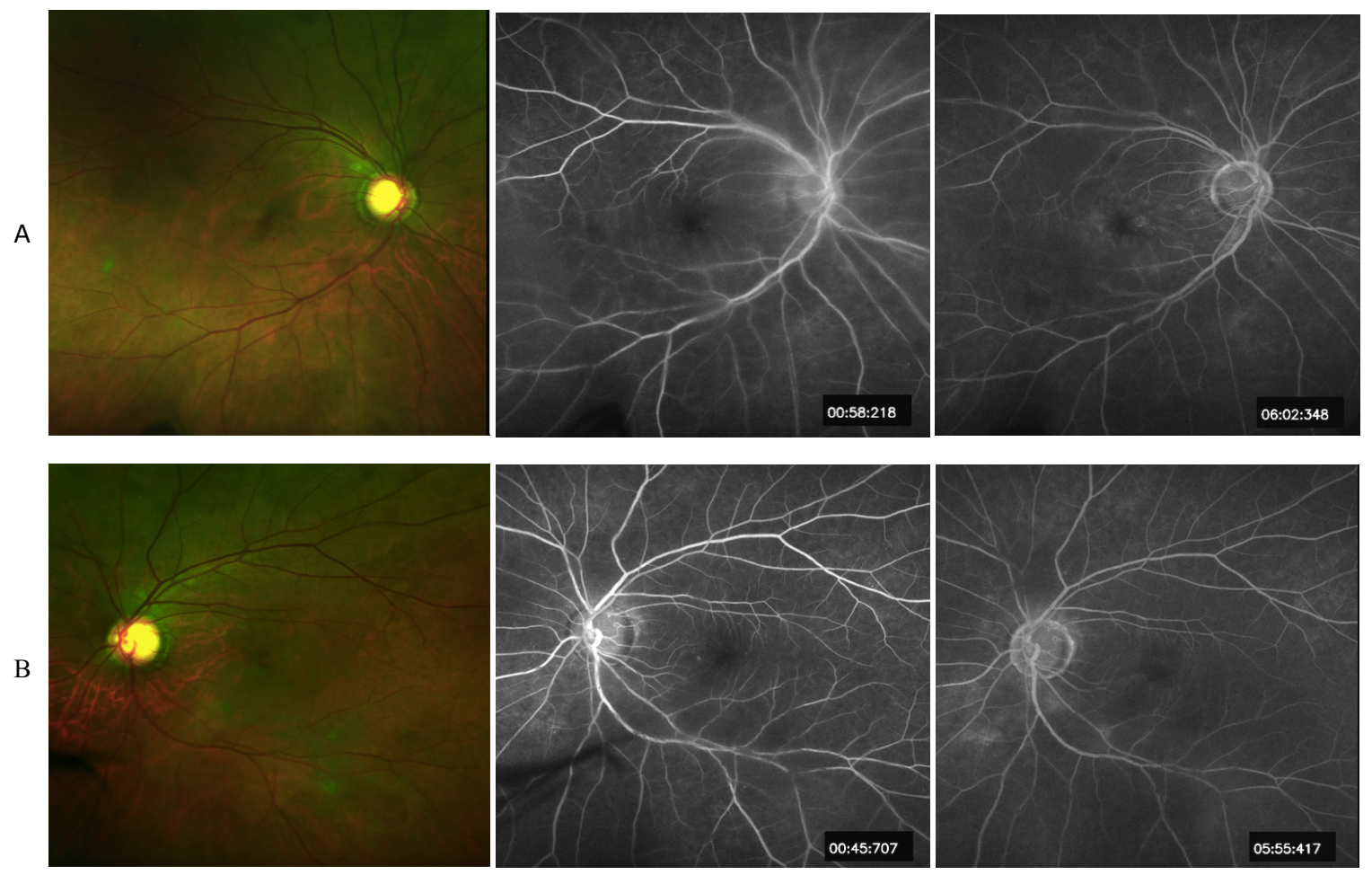

Figure 5. Optos Image with fluorescein angiography images A) Right eye B) Left eye. Both eyes demonstrated normal choroidal flush during early imaging with mild late leakage surrounding macula.

\section{Lab/Diagnostic Testing}

Although no lab testing is typically warranted for a first-time isolated anterior uveitis episode, due to macular edema secondary to possible spillover from anterior uveitis and availability of on-site lab testing at the VA, we deemed it necessary to carry out diagnostic lab testing. In private practice, lab testing is often costly and time-consuming. Therefore, it is important to try to narrow down the most pertinent tests based on case history and most common systemic etiologies based on whether the uveitis is anterior, posterior or intermediate. A review by Gutteridge \& Hall provides a good overview of what lab testing is indicated for several common conditions related to uveitis [10].

Our patient was carefully questioned in order to establish any possible systemic associations with uveitis. Lower back pain can be an indication of ankylosing spondylitis, especially in young males. Asking about any chest pain and prolonged cough can rule out tuberculosis. Furthermore, a history of oral/genital ulcers can indicate sarcoidosis and/or presence of herpes simplex virus. Gastrointestinal issues such as constipation can point to inflammatory bowel disease. Lyme disease is often found in people living in Northeast region with woodlands. Syphilis can manifest as a rash, fever, malaise, and/or joint pain.

Minimal lab testing should therefore include:

- Complete blood count (CBC)

- Urinalysis

- Lyme titers

- HLA-B27

- Antinuclear antibody (ANA)
- Angiotensin converting enzyme (ACE) test

- Venereal disease research laboratory (VDRL) test

- Fluorescent treponemal antibody absorption (FTA-ABS)

- Erythrocyte sedimentation rate (ESR)

- Chest X-ray if suspected tuberculosis and/or sarcoidosis

The lab testing for our patient determined he was HLA-B27 positive, which as stated above is the most common cause of nongranulomatous anterior uveitis. Due to high correlation of HLA-B27 positive patients with acute anterior uveitis and ankylosing spondylitis (AS) as well as the patient's history of arthritis, a SI joint fusion test was ordered. The SI joint fusion test requires a radiograph that images the sacroiliac joint. Ankylosing spondylitis tends to affect the axial skeleton, particularly the sacroiliac and spinal facet joints [11]. Therefore, radiography of the sacroiliac joint, near lower back, is often used as a diagnostic test for AS. In cases of AS, early signs include erosions on the iliac side of the joint followed by bony fusion of the sacroiliac joints, ultimately resulting in the classic "bamboo spine". At the end stage of AS, the sacroiliac joint may appear as a thin line or not be visible at all [12].

Although the SI joint fusion imaging did show mild spondylaropathy in our patient, no definitive diagnosis of ankylosing spondylitis was warranted. Therefore, the rheumatologist determined this acute event to be idiopathic in nature.

\section{Treatment and Management of Uveitis}

The standard therapy for acute anterior uveitis involves administering topical eye drops. The treatment usually includes topical 
corticosteroid drops such as prednisolone acetate $1 \%$ or Durezol $0.05 \%$ and a cycloplegic agent such as homatropine 5\% BID or scopolamine $0.25 \%$ TID.

Due to formulary restrictions when prescribing medication at the VA hospital, prednisolone acetate $1 \%$ presented the best choice. Durezol is often used in cases where prednisolone acetate $1 \%$ cannot quell the inflammation. Our patient was also started on cycloplegic medication BID OS which helped reduced pain as well as an adrenergic agonist, phenylephrine, to prevent posterior synechiae. Although based on AOA guidelines it is standard procedure to begin to taper the steroid dosage after at least two-fold reduction in anterior chamber reaction, due to HLA-B27 association and therefore increased risk of ocular complications, such as macular edema, as well as higher rates of recurrence, we decided to only begin tapering the prednisone acetate once the anterior chamber reaction was nearly resolved (trace cells) OS. The patient responded successfully to the medication, and inflammation was alleviated within a few follow-up visits.

Determining the cause of the macular edema: Uveitis $v s$. Diabetic retinopathy?

\section{Pathogenesis of CME}

Cystoid macular edema is an accumulation of fluid in the most central part of the retina and is usually the main threat to vision in the ocular disorders mentioned above [13]. The pathological disruption of the retinal vessels in the macular area, i.e. inner blood-retinal barrier (BRB), and/or dysfunction of retinal pigment epithelium (RPE), i.e. outer $\mathrm{BRB}$, is suggested to be the reason for fluid leakage. Intraocular inflammation, such as uveitis, may lead to disruption of both the inner and outer BRBs, in turn leading to fluid leakage into the retinal space. Specifically, cystoid spaces that form within Henle's layer demonstrate a loose arrangement allowing accumulation of fluid leakage from parafoveal capillaries [14].

\section{Management of CME}

Once CME is identified by SD-OCT, it is indicated to perform an IVFA to determine the source of the leakage. CME generally appears as a classic "flower petal" leakage pattern in late phase of IVFA [15]. However, the IVFA finding is dynamic and often difficult to interpret, especially with co-existing factors including retinal hemorrhages and exudates as well as leaky MAs. It is still important to use IVFA for assessment of foveal perfusion state in order to establish whether ischemia of the macula is present, which cannot be demonstrated with OCT.

\section{Treatment/Follow-up}

For clinically significant macular edema related to diabetic changes, two types of treatment are currently utilized: laser photocoagulation and anti-vascular endothelial growth factor (anti-VEGF) injections. Anti-VEGF therapy is the initial treatment choice for center-involving macular edema, with possible subsequent or deferred focal laser treatment. For non-center involving macular edema, many retina specialists prefer to use a modified ETDRS treatment approach [16]. This includes a less intense laser treatment, greater spacing, directly targeting MAs, and avoiding foveal vasculature within at least $500 \mu \mathrm{m}$ of the center of the macula [17]. In 2009, Scott et al. published a new study investigating the efficacy of focal/grid photocoagulation with non-center involved CSME. The authors found that although focal laser resulted in decreased fluorescein leakage after one year, visual acuity and retina thickness were relatively similar to eyes that did not receive any treatment [18].
Topical NSAIDs have been used off-label as a means to reduce macular edema related to uveitis. Studies have shown that use of nepafenac $0.1 \%$ improved mean outcome of visual acuity as well as reduced retinal thickness $[19,20]$. In severe cases, systemic oral steroids can be given and tapered according to clinical course; however, this is associated with well-known systemic side effects [21].

Anti-VEGF treatments are no longer being used only for CSME. In the past decade, new clinical trials have determined anti-VEGF injections to be equal if not superior to laser treatment in terms of final visual outcome and reduction in retinal thickness. In the recent VIBRANT study, results showed that aflibercept was more effective for managing macular edema associated with branch retinal vein occlusion compared to laser. The aflibercept group gained a mean of about 17 letters compared to seven letters in the laser group [22]. In a clinical trial performed by the Diabetic Retinopathy Clinical Research Network, aflibercept, bevacizumab, or ranizumab were all found to be effective in improving vision and reducing edema, with aflibercept only significantly superior at worse levels of initial visual acuity [23].

Furthermore, intravitreal implants of corticosteroids have proven in recent years to be a viable alternative to anti-VEGF in treating macular edema. Based on the FAME study, it was determined that the fluocinolone acetonide (FAc) intravitreal implant not only provides a similar benefit in terms of visual outcomes and reduction in edema, but it does so with a greatly reduced treatment burden for patients. In the FAME trials, patients received an average of 1.3 injections over a period of 3 years, whereas the anti-VEGF agents were administered monthly to bimonthly [24]. Still, there occur numerous side effects from corticosteroids including cataracts, elevated intraocular pressure and glaucoma. Therefore, anti-VEGF is still the initial treatment of choice for retinal edema.

In our case study, diclofenac was selected as initial treatment of choice due to size and location of the macular edema. This approach, however, was unsuccessful. Even so, the retinal specialist felt that neither anti-VEGF injections nor laser treatment were indicated at this time and that instead the area of edema warranted close observation every 3-4 months with OCT/photos to be evaluated at each follow-up visit.

\section{Foveal atrophy/macula ischemia secondary to uveitis and diabetic retinopathy}

Foveal atrophy can be a complication of intra-ocular inflammation related to uveitis, whether it is secondary to atrophy of RPE, CME, or macular ischemia secondary to occlusive retinal vasculitis [25]. Inflammatory damage to the RPE and choroid can cause dysfunction and atrophy of these tissues, leading to hypoxia and nutritional deprivation of the macula. Diabetes is well known to exert a negative effect on retinal vasculature, resulting in compromised capillaries leading to either edematous or ischemic changes. In a study by Channa et al. (2014) the authors found that patients with diabetic macula edema (DME) treated with ranibizumab who had foveal atrophy were more likely to have poorer visual outcome that those without foveal compromise [26].

OCT images of our patient demonstrated minimal disruption of photoreceptor integrity line (PIL) underneath the area of cystoid changes (Figure 2). Furthermore, IVFA did not show an enlarged foveal avascular zone and only minimal parafoveal capillary changes.

\section{Conclusion}

This case highlights the challenges of managing a patient with multiple systemic and ocular diseases and determining etiology of 
reduced vision. In these cases, a stepwise approach when diagnosing and treating the patient is warranted. We first sought to address the patient's chief complaint, which was pain/inflammation/irritation OS. Therefore, we performed all of the necessary ancillary and lab testing to determine the cause(s) of inflammation and treat promptly.

When trying to determine the source for reduced vision, it is necessary to work from the anterior part of the eye to the posterior. By performing $\mathrm{mPAM}$ we were able to eliminate confounding lenticular changes and determine the integrity of the macula itself. Since the patient was correctable to $20 / 20$ - vision with mPAM OS, it was confirmed that the reduced vision was not primarily related to macular edema, but rather cataracts.

Although edema was found on OCT, it is still important to obtain an IVFA which allows for the correlation of leakage with specific areas within the vasculature, in this case leaky MAs. Furthermore, the integrity of the PIL line on OCT and areas of capillary non-perfusion on IVFA must be considered, which can indicate long-term foveal atrophy. IVFA established the multiple MAs surrounding the fovea as cause of leakage OS. With elevated HbAlc to 15.4, a history of mild diabetic retinopathy with parafoveal MAs, and cystoid macular edema identified by OCT two years ago OU, we concluded that diabetic retinopathy was likely the etiology of macular edema OS. Although edema was not identified OD on SD-OCT, the inflammatory components from uveitis may have accelerated the leakage. It is important to note that in patients with inflammatory changes, such as macular edema, as well as multiple ocular disorders, the etiology is likely multifactorial.

Through extensive testing and treating each ocular condition in an orderly fashion, we were able to not only address and manage the patient's chief complaint, but also to determine the cause of macular edema, diabetic retinopathy, which will enable us to better manage the patient in the future. We can now focus on controlling blood sugar and following the patient every few months to monitor for resolution of macular edema.

Future follow-up exams are required to monitor edema and diabetic changes. If macular edema worsens, based on recent literature mentioned above, anti-VEGF or laser photocoagulation would constitute viable treatment options. Once blood sugar is controlled and edema resolves, we can be certain that the cataract OS is the source of reduced vision. Cataract surgery, however, cannot be performed for a few months following complete resolution of edema and inflammation.

\section{References}

1. Whitley W, Sheppard J. Lesson (2011) The Basics of Uveitis. Review of Optometry. Web. 10 March 2017.

2. Brewerton DA, Caffrey M, Nicholls A, Walters D, James DC (1973) Acute anterior uveitis and HL-A 27. Lancet 302: 994-996. [Crossref]

3. Linssen A, Rothova A, Valkenburg HA, Dekker-Saeys AJ, Luyendijk L, et al. (1991) The lifetime cumulative incidence of acute anterior uveitis in a normal population and its relation to ankylosing spondylitis and histocompatibility antigen HLA-B27. Invest Ophthalmol Vis Sci 32: 2568-2578. [Crossref]

4. Pulido JS, Streilein JW (2000) HLA and eye disease. In: Lechler R, Warrens A, (ed). HLA in Health and Disease, 2nd edn. San Diego: Academic Press 279-97.

5. Rosenbaum JT (1992) Acute anterior uveitis and spondyloarthropathies. Rheum Dis Clin North Am 18: 143-151. [Crossref]
6. Norn MS (1969) Endogenous uveitis. II. Recurrence rate. Acta Ophthalmol (Copenh) 47: 366-377. [Crossref]

7. Power WJ, Rodriguez A, Pedroza-Seres M, Foster CS (1998) Outcomes in anterior uveitis associated with the HLA-B27 haplotype. Ophthalmology 105: 1646-1651. [Crossref]

8. Fukuda S, Kogure M, Shimakawa M, Wakatsuki Y, Takahashi Y, et al. (1989) [Nongranulomatous anterior uveitis and HLA-B27 antigen]. Nippon Ganka Gakkai Zasshi 93: 412-417. [Crossref]

9. Rodriguez A, Akova YA, Pedroza-Seres M, Foster CS (1994) Posterior segment ocular manifestations in patients with HLA-B27-associated uveitis. Ophthalmology 101: 1267-1274. [Crossref]

10. Gutteridge IF, Hall AJ (2007) Acute anterior uveitis in primary care. Clin Exp Optom 90: 70-82. [Crossref]

11. Bennett DL, Ohashi K, El-Khoury GY (2004) Spondyloarthropathies: ankylosing spondylitis and psoriatic arthritis. Radiol Clin North Am 42: 121-134. [Crossref]

12. Akgul O, Ozgocmen S (2011) Classification criteria for spondyloarthropathies. World J Orthop 2: 107-115. [Crossref]

13. Lardenoye CW, van Kooij B, Rothova A (2006) Impact of macular edema on visual acuity in uveitis. Ophthalmology 113: 1446-1449. [Crossref]

14. Konstantinidis L, Berguiga M, Beknazar E, Wolfensberger TJ (2009) Anatomic and functional outcome after 23-gauge vitrectomy, peeling, and intravitreal triamcinolone for idiopathic macular epiretinal membrane. Retina 29: 1119-1127. [Crossref]

15. Jaffe G, Cahill MT (2006) Cystoid macular edema. In: Huang D (ed) Retinal Imaging, Mosby Elsevier 18: 206-218.

16. Early photocoagulation for diabetic retinopathy. ETDRS report number 9. Early Treatment Diabetic Retinopathy Study Research Group (1991) Ophthalmology 98: 766-785. [Crossref]

17. Fong DS, Strauber SF, Aiello LP, Beck RW, Callanan DG, et al. (2007) Writing Committee for the Diabetic Retinopathy Clinical Research Network. Comparison of the modified Early Treatment Diabetic Retinopathy Study and mild macular grid laser photocoagulation strategies for diabetic macular edema. Arch Ophthalmol 125: 469480. [Crossref]

18. Scott IU, Danis RP, Bressler SB, Bressler NM, Browning DJ, et al. (2009) Diabetic Retinopathy Clinical Research Network. Effect of focal/grid photocoagulation on visual acuity and retinal thickening in eyes with non-center-involved diabetic macular edema. Retina 29: 613-617. [Crossref]

19. Callanan D, Williams $P$ (2008) Topical nepafenac in the treatment of diabetic macular edema. Clin Ophthalmol 2: 689-692. [Crossref]

20. Hariprasad SM, Akduman L, Clever JA, Ober M, Recchia FM, et al. (2009) Treatment of cystoid macular edema with the new-generation NSAID nepafenac $0.1 \%$. Clin Ophthalmol 3: 147-154. [Crossref]

21. Rothova A, Suttorp-van Schulten MS, Frits Treffers W, Kijlstra A (1996) Causes and frequency of blindness in patients with intraocular inflammatory disease. $\mathrm{Br} J$ Ophthalmol 80: 332-336. [Crossref]

22. Campochiaro PA, Clark WL, Boyer DS, Heier JS, Brown DM, et al. (2015) Intravitreal aflibercept for macular edema following branch retinal vein occlusion: the 24-week results of the VIBRANT study. Ophthalmology 122: 538-544. [Crossref]

23. Wells JA, Glassman AR, Ayala AR, Jampol LM, Aiello LP, et al. (2015) Aflibercept bevacizumab, or ranibizumab for diabetic macular edema. N Engl J Med 372: 1193 1203. [Crossref]

24. Campochiaro PA, Hafiz G, Shah SM, Bloom S, Brown DM, et al. (2010) Famous Study Group. Sustained ocular delivery of fluocinolone acetonide by an intravitreal insert. Ophthalmology 117: 1393-1399. [Crossref]

25. Forooghian F, Yeh S, Faia LJ, Nussenblatt RB (2009) Uveitic foveal atrophy: clinica features and associations. Arch Ophthalmol 127: 179-186. [Crossref]

26. Channa R, Sophie R, Khwaja AA, Do DV, Hafiz G, Nguyen QD, et al.(2014) READ2 Study Group. Factors affecting visual outcomes in patients with diabetic macular edema treated with ranibizumab. Eye (Lond) 28: 269-278. [Crossref]

Copyright: (C2017 Goldberg LA. This is an open-access article distributed under the terms of the Creative Commons Attribution License, which permits unrestricted use, distribution, and reproduction in any medium, provided the original author and source are credited. 